\title{
Safety evaluation and consideration of 4 Pin Multi-needle for meso-therapy
}

\author{
Jun-Tae Kim ${ }^{\mathrm{a}}$, Ahnryul Choi ${ }^{\mathrm{a}}$, Jin-Hyoung Jeong ${ }^{\mathrm{a}}$, Jae-Hyun Jo ${ }^{\mathrm{a}}$, Ok-Su Ryu ${ }^{\mathrm{a}}$, Eun-Ji Kim ${ }^{\mathrm{b}}$, \\ Ki-Young Kim ${ }^{\mathrm{c}}$, Mi-Hee Song ${ }^{\mathrm{c}}$, Young-Ho Song ${ }^{\mathrm{c}}$, Woon-Seob Shin ${ }^{\mathrm{d}}$ and Sang-Sik Lee ${ }^{\mathrm{a}, *}$ \\ ${ }^{a}$ Department of Biomedical Engineering, Catholic Kwandong University, Gangneung, Korea \\ ${ }^{\mathrm{b}}$ Department of Healthcare Management, Catholic Kwandong University, Gangneung, Korea \\ ${ }^{\mathrm{c}}$ Department of Medical device R\&D Center, Hyundae Meditech Co., Ltd, Wonju, Korea \\ ${ }^{\mathrm{d}}$ Department of Microbiology, Catholic Kwandong University College of Medicine, Gangneung, Korea
}

\begin{abstract}
This study was conducted according to the method presented in the Republic of Korea Pharmacopoeia 11th Revision, aseptic test method to evaluate the suitability of sterilization for a sterile needle (4 Pin Multi-needle). In this study, four tests were conducted: sterility test, cytotoxicity test, acute toxicity test, skin sensitization test. First, in the aseptic test, the microorganism was not proliferated in the aseptic test of the medium. As a result of the performance test of the medium, it was confirmed that the microorganism developed within 3 days and the fungus was evident within 5 days. Based on this, it was confirmed that the medium was suitable, and as a result of the aseptic test, the development of microorganisms was not observed during the total culture period. Based on these results, tests were conducted which were confirmed to be suitable for aseptic testing because the development of bacteria on the provided samples was not recognized. For cytotoxicity tests ISO10993-5; 2009 (Biological Evaluation of Medical Devices, Part 5: Test for in vitro Cytotoxicity). As a result, the MEM eluate of the test substance caused very slight cytotoxicity to the fibroblasts of the mouse and was judged to be Grade 1 (Slightly cytotoxic) according to the judgment standard of ISO 10993-5. On the other hand, solvent control, negative control and positive control showed the expected results on the test. Acute Toxicity Test Results: It was judged that there was no systemic toxicity change when ICR mice were treated with $50 \mathrm{~mL} / \mathrm{kg} \mathrm{B}$.W. of the eluate of sterile injectable needle for 72 hours. Skin sensitization test result: The Hartley guinea pig was evaluated as a substance which is evaluated as a substance which does not induce any skin reaction when skin sensitization is applied to the dissected material of the sterile injectable needle and is weak in skin sensitivity. Based on the above tests, we will study the stability and efficacy of more reliable medical devices based on the verification and performance of medical devices.
\end{abstract}

Keywords: Meso-therapy, multi-Needle, medical device, safety evaluation

\section{Introduction}

Meso-therapy was developed by the French physician Pistor in 1952. It is a combination of 'meso' and 'therapy', which means healing, and it is mixed with proven medicines, minerals and vitamins. Injection therapy is injected only at the target site, unlike conventional therapy, so it can maximize the effect with a small amount and a minimum of pain and minimize the systemic side effects of the medicine or drug. Treatment subjects are variously applied to obesity, wrinkles, alopecia, and pain [1]. Drugs suitable purpose of treatment are mixed and injected with dozens to hundreds of injections depending

\footnotetext{
${ }^{*}$ Corresponding author: Sang-Sik Lee, Department of Biomedical Engineering, Catholic Kwandong University, Gangneung, Korea. E-mail: lsskyj@cku.ac.kr.
}

0928-7329/18/\$35.00 (C) 2018 - IOS Press and the authors. All rights reserved

This article is published online with Open Access and distributed under the terms of the Creative Commons Attribution NonCommercial License (CC BY-NC 4.0). 
on the treatment site using insulin sensitizer or automatic syringe. The frequency of treatment is usually $1 \sim 2$ weeks apart, depending on the response to treatment at $1 \sim 2$ months intervals to widen the treatment interval and maintenance treatment is done once every $4 \sim 5$ months.

It is a new treatment technology widely applied in 14 countries including Europe, America and some Asian countries.

Using meso-therapy, collagen in the dermal layer of the skin is reduced and denatured to reduce the elasticity and wrinkles caused by the dullness of the skin, thereby promoting healthy collagen production in the dermis without damaging the surface of the skin through the treatment, thereby improving dull and wrinkled skin. Minimal amounts of vitamins and antioxidants are injected directly into the skin to maximize skin regeneration [2-5].

In combination with a drug that activates blood circulation and lymphatic circulation and a drug that breaks down fat, it can accelerate the decomposition of cellulite using a procedure in a thick cellulite on the side of the hips or thighs.

Vitamin C, antioxidants, decolorizing agents, etc., are added to the skin that looks dark due to the melanin pigment, and it can be treated by injecting it into the epidermis and dermis of the skin. And meso-therapy is more effective than any treatment method on scalp (hair loss) [6]. By injecting drugs with scalp health and hair growth effect directly into the hair follicles, it helps to shorten hair loss period and help hair grow quickly. It is a more effective treatment if you use ancillary interactions with existing hair loss treatments. A syringe used for meso-therapy usually uses a 1 pin-needle syringe. Due to the nature of the procedure, the practitioner who is performing the injection in tens to hundreds of places feels a lot of fatigue.

The 4 Pin Multi-needle was developed to enhance the convenience and efficiency of the practitioner and safety of the device could be secured through safety evaluation, which would improve the efficiency and convenience of the practitioner in meso-therapy.

The present study is expected to improve the safety and safety of the procedure from the safety factors of infection and other factors through the safety evaluation of 4 Pin Multi-needle made for use in mesotherapy.

\section{Materials and methods}

For the safety evaluation of 4 Pin Multi-needle for meso-therapy, tests such as aseptic test, cytotoxicity test, acute toxicity test, skin sensitization test were conducted. For the four tests, it is the safety evaluation of the medical device conforming to the CE certification [7-15]. The developed 4 Pin Multi-needle is able to inject drugs more effectively into the skin using mesotherapy.

In addition, it is possible to shorten the procedure and administration time by using four needles. In other words, it can be used effectively by the reduction of time and costs.

\subsection{Aseptic test}

A medical device is a device or device used for a person or an animal with the purpose of treating a disease or the like, and should not be toxic when applied to the body. In addition, medical devices must remain sterile until used to prevent secondary infection by microbial contamination. Sterile testing is widely used as a means of evaluating the suitability of sterilization for a variety of medical devices and test materials. This test was carried out in order to evaluate the proliferation of microorganisms (bacteria and fungi) that were proliferated when the test substances were directly inoculated into liquid thioglycolic acid medium and soybean casein digest medium and cultured at each temperature. 
Table 1

Strain for medium performance test

\begin{tabular}{lll}
\hline Culture medium & Test strains & Culture \\
\hline Liquid thioglycolic acid medium & Staphylococcus aureus (ATCC 6538) & $(30 \sim 35)^{\circ} \mathrm{C}$, aerobic culture \\
& $\begin{array}{l}\text { Pseudomonas aeruginosa (ATCC 9027) } \\
\text { Clostridium sporogenes (ATCC 11437) }\end{array}$ & \\
Soybean casein digestion medium & $\begin{array}{l}\text { Bacillus subtilis (ATCC 6633) } \\
\text { Candida albicans (ATCC 10231) }\end{array}$ & $\left(\right.$ 20 25) ${ }^{\circ} \mathrm{C}$, aerobic culture \\
& Aspergillus brasiliensis (ATCC 16404) & \\
\hline
\end{tabular}

\subsubsection{Aseptic test materials and methods}

1) Test substance: Sterile needle (4 Pin Multi-needle).

2) Aseptic properties of medium.

(1) Aseptic properties of medium: Microbial growth should not occur when the liquid thioglycolic acid medium is cultured at $(30 \sim 35)^{\circ} \mathrm{C}$ and soybean casein digestion medium is cultured at $(20 \sim 25)^{\circ} \mathrm{C}$ for 14 days.

(2) Performance test of the medium: When the culture medium of the following performance test strains or the strains considered to be equivalent thereto is inoculated at a rate of not more than $100 \mathrm{CFU}$ per 1 culture medium, each bacterium must develop clearly within 5 days.

3) Aseptic test (direct method).

(1) Test method.

All or part of the specimen was inoculated into the liquid thioglycolic acid medium and the soybean casein digestion medium, respectively. Liquid thioglycolic acid medium $(30 \sim 35)^{\circ} \mathrm{C}$ and soybean casein digestion medium were cultured at $(20 \sim 25)^{\circ} \mathrm{C}$ for 14 days, and the growth of bacteria was observed at 5 9 and 14 days after culturing. If the medium is turbid depending on the specimen, or if it is otherwise difficult to determine, or if necessary, the appropriate amount is transplanted into the new medium on the 14th day of culture and cultured for 4 days or more with the original medium at the same temperature.

\subsection{Cytotoxicity test}

In vitro cytotoxicity testing is commonly used as an early means of assessing biocompatibility for a variety of medical devices and test materials because of the relatively easy access to the test. This test was conducted to evaluate whether the test substance causes cytotoxicity in mouse fibroblasts (NCTC Clone 929 (L-929)) under the culture conditions of the cells. According to ISO 10993-5: 2009 (Part 5: Tests for in vitro cytotoxicity), in order to apply a solid test substance to a cell, it was eluted with MEM medium supplemented with $10 \%$ serum A test solution was prepared. This test solution was directly contacted with the cells to examine the effect of the test substance eluate on the cells under a microscope. The MEM medium supplemented with $10 \%$ serum used as the elution solvent was selected to maintain the physiological condition most similar to the situation in which the test substance was applied to the human body.

\subsubsection{Cytotoxicity test (materials and methods)}

1) Test substance: Sterile needle (4 Pin Multi-needle).

2) Medium and vehicle.

- Appellation: $1 \times$ MEM (Minimum essential medium) with $10 \%$ serum; 
Table 2

ISO 10993-5, 8.5 Determination of cytotoxicity

\begin{tabular}{cll}
\hline Grade & Reactivity & Conditions of all cultures \\
\hline 0 & None & $\begin{array}{l}\text { Discrete intracytoplasmatic granules, no cell lysis, no reduction of cell growth } \\
\text { Not more than 20\% of the cells are round, loosely attached and without intracytoplasmic granules, or } \\
\text { show changes in morphology; occasional lysed cells are present; only slight growth inhibition observable }\end{array}$ \\
2 & Slight & $\begin{array}{l}\text { Not more than 50\% of the cells are round, devoid of intracytoplasmic granules, no extensive cell lysis; } \\
\text { not more then 50\% growth inhibition observable }\end{array}$ \\
3 & Moderate & $\begin{array}{l}\text { Not more than 70\% of the cell layers contain rounded cells or are lysed; cell layers not completely } \\
\text { destroyed, but more than 50\% growth inhibition observable }\end{array}$ \\
4 & Severe & Nearly complete or complete destruction of the cell layers \\
\hline
\end{tabular}

- Manufacturer: Gibco;

- Lot No.: 1687974;

- Conditions for keeping: $(2 \sim 8)^{\circ} \mathrm{C}$.

3) Serum.

- Appellation: Horse serum;

- Manufacturer: Gibco;

- Lot No.: 1517711;

- Conditions for keeping: $(-15 \sim-20)^{\circ} \mathrm{C}$.

4) Negative control.

- Appellation: High density polyethylene Film;

- Manufacturer: Hatano Research Institute (Food and Drug Safety Center);

- Lot No.: C-111;

- Conditions for keeping: Room temperature.

5) Positive control.

- Appellation: ZDEC Polyurethan Film;

- Manufacturer: Hatano Research Institute (Food and Drug safety Center);

- Lot No.: A-141;

- Conditions for keeping: Room temperature.

6) Preparation of experimental material: after aseptically operating at a rate of $20 \mathrm{~mL}$ of $4 \mathrm{~g} 10 \%$ MEM medium supplemented with serum per test substance, the cells were eluted in a $(5+1) \%$ $\mathrm{CO}_{2}$ incubator at $(37 \pm 1)$ This solution was used as a test solution. For the solvent control, the MEM medium except for the test substance was eluted under the same elution conditions as the test substance. The negative control and the positive control were assayed in accordance with the cytotoxicity test method of the manufacturer (Hatano Research Institute) at a rate of $10 \mathrm{~mL}$ of $1 \times$ MEM medium per $1 \mathrm{~g}(37 \pm 1)^{\circ} \mathrm{C}$. $(24 \pm 2)$ hours in a $(5+1) \% \mathrm{CO}_{2}$ incubator. The test substance was used for the test within 24 hours after preparation.

7) Test system.

(1) Test cell line.

- Appellation: NCTC Clone 929 (L-929);

- Manufacturer: ATCC (American Type Culture Collection, USA).

(2) Reason for selecting test system: These cells are known to be highly susceptible to chemicals in cytotoxicity tests and were selected because of the abundance of data related to cytotoxicity studies. 
Table 3

Test group composition

\begin{tabular}{lclcl}
\hline Group & Animal number & Test materials & Dose (mL/kg B.W.) & Injection site \\
\hline G1 negative control & $1101 \sim 1105$ & Sterile saline & 50 & Intravenous \\
G2 treatment & $1201 \sim 1205$ & Sterile saline extract & 50 & \\
G3 negative control & $1301 \sim 1305$ & Cotton seed oil & 50 & Intraperitoneal \\
G4 treatment & $1401 \sim 1405$ & Cotton seed oil extract & 50 & \\
\hline
\end{tabular}

Table 4

Test group composition

\begin{tabular}{lcll}
\hline Group & Animal number & Test materials dose (mL/kg B.W.) injection site \\
\hline G1 negative control & $1101 \sim 1105$ & Negative control (sterile saline) & Test materials (sterile saline extract) \\
G2 treatment & $1201 \sim 1210$ & Test materials (sterile saline extract) & Test materials (sterile saline extract) \\
G3 negative control & $1301 \sim 1305$ & Negative control (cotton seed oil) & Test materials (cotton seed oil extract) \\
G4 treatment & $1401 \sim 1410$ & Test materials (cotton seed oil extract) & Test materials (cotton seed oil extract) \\
\hline
\end{tabular}

(3) Culture conditions.

Culture medium: 10\% Horse serum and Antibiotics [Penicillin (100 Units/mL), Streptomycin $(100 \mathrm{ug} / \mathrm{mL})$ include with Minimum Essential Medium (pH 7.4) Condition: $(5 \pm 1) \% \mathrm{CO}_{2}$ and $(37 \pm 1)^{\circ} \mathrm{C}$ were transplanted every 3 to 4 days.

8) Test methods.

(1) Qualitative analysis.

The monolayer cultured cells were treated with trypsin (EDTA) to adjust the cell concentration to 105 per $1 \mathrm{~mL}$, and inoculated $2 \mathrm{~mL}$ into a $10-\mathrm{cm} 26$-well plate $(35 \mathrm{~mm} / \mathrm{well})$. The wells were cultured for 24 hours, monolayer cultures were selected, labeled with each test group and control group, and then the medium was removed. $(5 \pm 1) \% \mathrm{CO}_{2},(37 \pm 1)^{\circ} \mathrm{C}$, and $2 \mathrm{~mL}$ of the test substance effluent, solvent control, negative control and positive control in 3 selected wells in cultured for 48 hours. After incubation, the dissolution or morphology of (100x) cells was observed under a microscope.

(2) Quantitative analysis.

After observing the dissolution and morphology of the cells, trypsin (EDTA) was applied to remove the cells from the plate, and the viable cells in each test group were counted.

9) Judgment of cytotoxicity.

$(+)$ In the presence of a uniform monolayer of cells (Confluent monolayer), or (-) in the absence of a single monolayer of cells. The cell solubility and morphological changes of each well were recorded. The color of the medium of the negative control group and the test group was also observed. When the color of the medium changed to yellow, it was judged that the medium was changed to acid by the eluate. When the color changed to magenta or purple, it was judged that the medium changed to basic.

To demonstrate the efficacy of the test, the solvent control (Reagent control) and negative control should not show cytotoxicity (Grade 0 ) and positive control should show moderate cytotoxicity (> Grade 2).

If each control does not show the expected result, or if different results are observed in the three test groups, retest is performed.

\subsection{Acute toxicity test}

To investigate the acute toxicity of sterile injectable needles, intravenous and intraperitoneal doses of 
Table 5

Cranial end

\begin{tabular}{|c|c|c|c|c|c|}
\hline \multicolumn{6}{|c|}{ Cranial end } \\
\hline (1) & $\mathbf{\square}$ & $(0.1 \mathrm{~mL})$ & $(0.1 \mathrm{~mL})$ & [ & (1) \\
\hline (2) & $\mathbf{\square}$ & $(0.1 \mathrm{~mL})$ & $(0.1 \mathrm{~mL})$ & 口 & (2) \\
\hline (3) & $\mathbf{\square}$ & $(0.1 \mathrm{~mL})$ & $(0.1 \mathrm{~mL})$ & [ & (3) \\
\hline
\end{tabular}

sterile saline and cottonseed oil were administered intravenously and intraperitoneally to ICR mice at a dose of $50 \mathrm{~mL} / \mathrm{kg} \mathrm{BW}$. Mortality, general symptoms, weight changes and toxicity respectively.

During the test, no general symptoms, no specific clinical symptoms and no deaths were observed.

The body weight was measured during the test period, and all the body weight changes were normal.

No specific biological response was observed for all animals when observed for 72 hours after administration of the test substance.

From the above results, it was judged that the systemic toxicity change was not observed when ICR mice were treated with $50 \mathrm{~mL} / \mathrm{kg} \mathrm{B}$.W. of the sterile injectable needle for 72 hours.

\subsubsection{Animal ethics}

This test is based on the Animal Protection Act [Law No. 13023 (2015-01-20, partly revised)] and the Laboratory Animals Act [Act No. 11987 (2013-07-30, partly revised)]. It was approved by the Animal Ethics Committee of Health Care Headquarters.

\subsubsection{Acute toxicity test (materials and methods)}

1) Test substance: Sterile needle (4 Pin Multi-needle).

2) Preparation of test materials: Sterile physiological saline (Daihan Pharm CO., LTD, Korea) and cottonseed oil (Junsei Chemical CO., LTD, Japan) were prepared at a temperature of $70^{\circ} \mathrm{C}$ to 24 hours at a ratio of $20 \mathrm{~mL}$ per $4 \mathrm{~g}$ of the weight of the test substance, which was used as a test substance. Separately, only sterile physiological saline and cottonseed oil were used as negative control substances by reacting under the same conditions.

3) Animal Model information and condition.

(1) Animal species: Mouse (CrljOri: CD1 (ICR)).

(2) The weight range of inject with Syringe, Number of animals used and weeks of age: (17-23 g), Number of 20 (male), 4 weeks old.

(3) Reason for selection: The ICR mouse used in this study is a species commonly used in acute toxicity tests and has a relatively rich test base data, so it is easy to interpret and evaluate the test results.

(4) Purification and Quarantine: After the introduction of the animals, the animals were quarantined and purified under the environment of the animal breeding room of the researcher and the general health condition was observed, and healthy individuals were selected for the test.

(5) Breeding environment.

A. Environmental condition: This test was conducted under the conditions of temperature (22 $\pm 3)^{\circ} \mathrm{C}$, relative humidity $(50 \pm 20) \%$, number of ventilation $(10 \sim 20)$ times $/ \mathrm{hr}$, lighting time 12 hours (08:00 20:00) Lux was raised in the animal breeding room of the present researcher.

B. Breeding condition: Five animals were housed in Polysulfonate cage [180 (W) $\mathrm{mm} \times$ $300(\mathrm{D}) \mathrm{mm} \times 140(\mathrm{H}) \mathrm{mm}$, ThreeShine, Korea] during the refinement and the test period.

C. Rodent Diet 205053 [Labdiet, USA], irradiated sterilized feed and feed water, and free $\mathrm{R} / \mathrm{O}$ water were used for negative feed. 
Table 6

Caudal end

\begin{tabular}{|c|c|c|c|c|}
\hline Group & Animal number & Test materials & Dose $(\mathrm{mL})$ & Injection site \\
\hline G1 negative control & $1101 \sim 1105$ & $\begin{array}{l}\text { Sterile saline: FCA }(1: 1) \\
\text { Sterile saline: FCA }(1: 1) \\
\text { Blank (Sterile saline) } \\
\text { Blank (Sterile saline) } \\
\text { Blank (Sterile saline): FCA }(1: 1) \\
\text { Blank (Sterile saline): FCA }(1: 1)\end{array}$ & $\begin{array}{l}0.1 \\
0.1 \\
0.1 \\
0.1 \\
0.1 \\
0.1\end{array}$ & $\begin{array}{l}\text { (1) } \\
\text { (1) } \\
\text { (2) } \\
\text { (2) } \\
\text { (3) } \\
\text { (3) }\end{array}$ \\
\hline G2 treatment & $1201 \sim 1210$ & $\begin{array}{l}\text { Sterile saline: FCA }(1: 1) \\
\text { Sterile saline: FCA }(1: 1) \\
\text { Sterile saline extract } \\
\text { Sterile saline extract } \\
\text { Sterile saline extract: FCA }(1: 1) \\
\text { Sterile saline extract: FCA }(1: 1)\end{array}$ & $\begin{array}{l}0.1 \\
0.1 \\
0.1 \\
0.1 \\
0.1 \\
0.1\end{array}$ & $\begin{array}{l}1 \\
\text { (1) } \\
\text { (2) } \\
\text { (2) } \\
\text { (3) } \\
\text { (3) }\end{array}$ \\
\hline G3 negative control & $1301 \sim 1305$ & $\begin{array}{l}\text { Cotton seed oil: FCA (1:1) } \\
\text { Cotton seed oil: FCA (1:1) } \\
\text { Blank (Cotton seed oil) } \\
\text { Blank (Cotton seed oil) } \\
\text { Blank (Cotton seed oil): FCA }(1: 1) \\
\text { Blank (Cotton seed oil): FCA }(1: 1)\end{array}$ & $\begin{array}{l}0.1 \\
0.1 \\
0.1 \\
0.1 \\
0.1 \\
0.1\end{array}$ & $\begin{array}{l}\text { (1) } \\
\text { (1) } \\
\text { (2) } \\
\text { (2) } \\
\text { (3) } \\
\text { (3) }\end{array}$ \\
\hline G4 treatment & $1401 \sim 1410$ & $\begin{array}{l}\text { Cotton seed oil: FCA }(1: 1) \\
\text { Cotton seed oil: FCA }(1: 1) \\
\text { Cotton seed oil extract } \\
\text { Cotton seed oil extract } \\
\text { Cotton seed oil extract: FCA }(1: 1) \\
\text { Cotton seed oil extract: FCA }(1: 1)\end{array}$ & $\begin{array}{l}0.1 \\
0.1 \\
0.1 \\
0.1 \\
0.1 \\
0.1\end{array}$ & $\begin{array}{l}1 \\
\text { (1) } \\
\text { (2) } \\
\text { (2) } \\
\text { (3) } \\
\text { (3) }\end{array}$ \\
\hline
\end{tabular}

FCA : Freund's complete adjuvant.

4) Configuration and individual identification of test groups.

- Object identification: The individual identification was indicated by the individual number using the oil magic on the tail and the animal identification card with the test number, sex, group number, individual number, test period and the person in charge of the test was attached to the breeding box.

5) Test methods.

Measurement of the body weight of each mouse before administration of tablets, vein using a disposable syringe (26 Gauge) with 5 mice $50 \mathrm{~mL} / \mathrm{kg}$ B.W. the control substance and the test substance in (sterile saline eluate) and intraperitoneal (cotton seed oil eluate).

6) Progress observation.

(1) General symptom observation: All animals were observed for changes of general symptoms and presence of dead animals at 4, 24, 48 and 72 hours after injection of the test substance.

(2) Weight measurement: Body weight was measured at the time of introduction, on the day of administration and at 24, 48 and 72 hours after administration.

7) Assessment methods.

If the test group shows no significant biological response compared to the control group for 72 hours after injection of the test substance, it is considered that it does not show acute toxicity. Acute toxicity is also seen when two or more animals die in the test group, abnormal behavior such as seizures or debilitation occurs in two or more animals, or weight loss of more than $10 \%$ in three or more animals. If less than one animal exhibits an overall symptom of a biological response or dies, the test is repeated using 10 animal groups. In the repeated tests, 10 animals treated 
Table 7

Magnuson and Krigman grade table

\begin{tabular}{lc} 
Patch test reaction & Grade \\
\hline No noticeable change & 0 \\
Separable or refractory erythema & 1 \\
Moderate erythema and fusiform erythema & 2 \\
Severe erythema and swelling & 3 \\
\hline
\end{tabular}

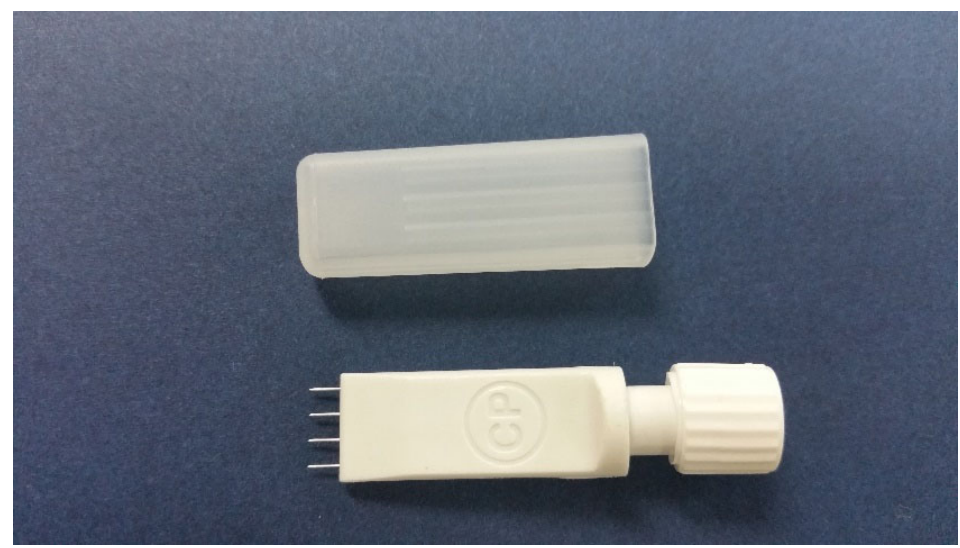

Fig. 1. Four pin-multi needle for Meso-therapy.

with the test substance are considered not to show acute toxicity if there is no specific biological response than the control.

\subsection{Skin sensitization test (materials and methods)}

In order to investigate the skin sensitization to the sterile injectable needle, the Hartley guinea pig was induced to induce sensitization by primary intradermal injection and second transdermal application of sterilized physiological saline and cottonseed oil, and then transdermal application to induce mortality, general symptoms and skin sensitization.

A. During the test, no general symptoms, no specific clinical symptoms and no deaths were observed.

B. The body weight was measured during the test period, and all the body weight changes were normal.

C. After skin sensitization, 24 hours and 48 hours of skin reactions were evaluated. No skin reactions such as erythema and edema were observed.

As a result, it was evaluated that Hartley guinea pigs did not induce any skin reaction when exposed to sensitization of the sterile injectable needle.

\subsubsection{Animal ethics}

This test is based on the Animal Protection Act [Law No. 13023 (2015-01-20, partly revised)] and the Laboratory Animals Act [Act No. 11987 (2013-07-30, partly revised)]. It was approved by the Animal Ethics Committee of Health Care Headquarters.

1) Test substance: Sterile needle.

2) Preparation of test materials: Sterile physiological saline (Daihan Pharm CO., LTD, Korea) and 
Table 8

Aseptic testing of media

\begin{tabular}{lcl}
\hline Culture medium & Culture confirmation & Judgment \\
\hline Liquid thioglycolic acid medium & N.G. & Suitable \\
Soybean casein digestion medium & N.G. & Suitable \\
\hline
\end{tabular}

*G.: Growth, N.G.: No growth.

Table 9

Performance tests of the culture medium

\begin{tabular}{llcll}
\hline Culture medium & Test strains & $\begin{array}{c}\text { Inoculation } \\
\text { amount }(\mathrm{CFU})\end{array}$ & Culture & Judgment \\
\hline Liquid thioglycolic acid culture medium & S. aureus & $\leqslant 100$ & Exhalation culture $(30 \sim 35)^{\circ} \mathrm{C}, 3$ days & G. \\
& P. sporogenes & $\leqslant 100$ & Exhalation culture $(30 \sim 35)^{\circ} \mathrm{C}, 3$ days & G. \\
& C. soirigenes & $\leqslant 100$ & Exhalation culture $(30 \sim 35)^{\circ} \mathrm{C}, 3$ days & G. \\
Soybean casein digestion culturemedium & B. subtilis & $\leqslant 100$ & Exhalation culture $(20 \sim 25)^{\circ} \mathrm{C}, 3$ days & G. \\
& C. albicans & $\leqslant 100$ & Exhalation culture $(20 \sim 25)^{\circ} \mathrm{C}, 3$ days & G. \\
& A. brasiliensis & $\leqslant 100$ & Exhalation culture $(20 \sim 25)^{\circ} \mathrm{C}, 3$ days & G. \\
\hline
\end{tabular}

*G.: Growth, N.G.: No growth.

Table 10

Aseptic test

\begin{tabular}{clcccc}
\hline \multicolumn{2}{c}{ Culture medium } & Exp start date & Intermediate inspection day & Last inspection day & Judgment \\
\hline Liquid thioglycolic & Specimen & - & N.G. & N.G. & Suitable \\
acid culture medium & Negative control & - & N.G. & N.G. & \\
$\begin{array}{c}\text { Soybean casein digestion } \\
\text { culturemedium }\end{array}$ & Specimen & - & N.G. & N.G. & Suitable \\
\hline
\end{tabular}

*G.: Growth, N.G.: No growth.

Table 11

Result of qualitative analysis

\begin{tabular}{|c|c|c|c|c|c|c|c|}
\hline Well & $\begin{array}{l}\text { Confluent } \\
\text { monolayer }\end{array}$ & $\begin{array}{l}\% \text { Growth } \\
\text { inhibition }\end{array}$ & $\begin{array}{c}\% \text { Cells without } \\
\text { intracellular granulation }\end{array}$ & \% Rounding & \% Lysis & Reactivity & Grade \\
\hline Test (1) & $(+)$ & 10 & 10 & 10 & 10 & Slight & 1 \\
\hline Test (2) & $(+)$ & 10 & 10 & 10 & 10 & Slight & 1 \\
\hline Test (3) & $(+)$ & 10 & 10 & 10 & 10 & Slight & 1 \\
\hline Negative control (1) & $(+)$ & 0 & 0 & 0 & 0 & None & 0 \\
\hline Negative control (2) & $(+)$ & 0 & 0 & 0 & 0 & None & 0 \\
\hline Negative control (3) & $(+)$ & 0 & 0 & 0 & 0 & None & 0 \\
\hline Reagent control (1) & $(+)$ & 0 & 0 & 0 & 0 & None & 0 \\
\hline Reagent control (2) & $(+)$ & 0 & 0 & 0 & 0 & None & 0 \\
\hline Reagent control (3) & $(+)$ & 0 & 0 & 0 & 0 & None & 0 \\
\hline Positive control (1) & $(+)$ & 100 & 100 & 100 & 100 & Severe & 4 \\
\hline Positive control (2) & $(+)$ & 100 & 100 & 100 & 100 & Severe & 4 \\
\hline Positive control (3) & $(+)$ & 100 & 100 & 100 & 100 & Severe & 4 \\
\hline
\end{tabular}

$(+)$ : Present, $(-)$ : Absent.

cottonseed oil (Junsei Chemical CO., LTD, Japan) were prepared at a temperature of $70^{\circ} \mathrm{C}$ to 24 hours at a ratio of $20 \mathrm{~mL}$ per $4 \mathrm{~g}$ of the weight of the test substance, which was used as a test substance. Separately, only sterile physiological saline and cottonseed oil were used as negative control substances by reacting under the same conditions.

3) Animal model information and condition. 
Table 12

Result of qualitative analysis

\begin{tabular}{ccccc}
\hline \multirow{2}{*}{ Group line } & \multicolumn{4}{c}{ Result of cell counting (cells/mL) } \\
\cline { 2 - 4 } & Test group & Reagent control & Negative control & Positive control \\
\hline 1 & $6.6 \times 10^{5}$ & $7.8 \times 10^{5}$ & $7.6 \times 10^{5}$ & 0 \\
2 & $6.9 \times 10^{5}$ & $7.5 \times 10^{5}$ & $7.7 \times 10^{5}$ & 0 \\
3 & $6.5 \times 10^{5}$ & $7.7 \times 10^{5}$ & $7.4 \times 10^{5}$ & 0 \\
Average & $6.7 \times 10^{5}$ & $7.7 \times 10^{5}$ & $7.6 \times 10^{5}$ & 0 \\
RCC $^{*}(\%)$ & 87.0 & 100.0 & 98.7 & 0.0 \\
\hline
\end{tabular}

RCC (relative cell counting, \%) = Cell number of test group/Cell number of reagent control group $\times 100$.

Table 13

Mortality and clinical signs

\begin{tabular}{|c|c|c|c|c|c|c|c|}
\hline \multirow[t]{2}{*}{ Group } & \multirow[t]{2}{*}{ Sex } & \multirow[t]{2}{*}{ Animal number } & \multicolumn{4}{|c|}{ Clinical signs } & \multirow[t]{2}{*}{ Mortality (dead/total) } \\
\hline & & & $4 \mathrm{~h}$ & $24 \mathrm{~h}$ & $48 \mathrm{~h}$ & $72 \mathrm{~h}$ & \\
\hline \multirow[t]{5}{*}{ G1 negative control (sterile saline) } & Male & 1101 & - & - & - & - & $0 / 5^{\mathrm{a}}(0 \%)$ \\
\hline & & 1102 & - & - & - & - & \\
\hline & & 1103 & - & - & - & - & \\
\hline & & 1104 & - & - & - & - & \\
\hline & & 1105 & - & - & - & - & \\
\hline \multirow[t]{5}{*}{ G2 treatment (sterile saline extract) } & Male & 1201 & - & - & - & - & $0 / 5(0 \%)$ \\
\hline & & 1202 & - & - & - & - & \\
\hline & & 1203 & - & - & - & - & \\
\hline & & 1204 & - & - & - & - & \\
\hline & & 1205 & - & - & - & - & \\
\hline \multirow[t]{5}{*}{ G3 negative control (cotton seed oil) } & Male & 1301 & - & - & - & - & $0 / 5(0 \%)$ \\
\hline & & 1302 & - & - & - & - & \\
\hline & & 1303 & - & - & - & - & \\
\hline & & 1304 & - & - & - & - & \\
\hline & & 1305 & - & - & - & - & \\
\hline \multirow[t]{5}{*}{ G4 treatment (cotton seed oil extract) } & Mael & 1401 & - & - & - & - & $0 / 5(0 \%)$ \\
\hline & & 1402 & - & - & - & - & \\
\hline & & 1403 & - & - & - & - & \\
\hline & & 1404 & - & - & - & - & \\
\hline & & 1405 & - & - & - & - & \\
\hline
\end{tabular}

-: No abnormality detected, ${ }^{\mathrm{a}}$ : No. of dead animals/No. of total animals.

(1) Animal species: Guinea pig, SPF.

(2) The weight range of inject with Syringe, Number of animals used and weeks of age: (300$500 \mathrm{~g}$ ), Number of 30 (female), 6 weeks old.

(3) Reason for selection: The guinea pigs used in this study were selected because they were generally used for skin sensitization tests and had a relatively rich test basis data, which made it easy to interpret and evaluate the test results.

(4) Purification and quarantine: After the introduction of the animals, the animals were quarantined under the environment of the animal breeding room of the researcher, and the general health condition was observed while being purified, and healthy individuals were selected for the test.

4) Breeding environment.

(1) Environmental condition: This test was conducted under the conditions of temperature (20 $\pm 3)^{\circ} \mathrm{C}$, relative humidity $(50 \pm 20) \%$, number of ventilation (10 20) times/hr, lighting time 12 hours (08:00 20:00)) Lux was raised in the animal breeding room of the present researcher. 
Table 14

Body weights (SEX: Male, Unit: g)

\begin{tabular}{lcrr}
\hline Group & Animal number & \multicolumn{2}{c}{ Day(s) after injection } \\
\cline { 3 - 4 } & & 0 day & 3 days \\
\hline G1 negative control (sterile saline) & 1101 & 18.08 & 22.78 \\
& 1102 & 18.56 & 24.43 \\
& 1103 & 19.36 & 24.00 \\
& 1104 & 19.56 & 24.82 \\
G2 treatment (sterile saline extract) & 1105 & 18.02 & 23.89 \\
& Mean & 18.72 & 23.98 \\
& S.D. & 0.71 & 0.77 \\
& 1201 & 18.17 & 22.81 \\
& 1202 & 18.33 & 23.05 \\
G3 negative control (cotton seed oil) & 1203 & 17.77 & 21.84 \\
& 1204 & 17.90 & 21.78 \\
& 1205 & 20.54 & 25.59 \\
& Mean & 18.54 & 23.01 \\
& S.D. & 1.13 & 1.55 \\
& 1301 & 18.50 & 23.62 \\
& 1302 & 17.85 & 22.15 \\
G4 treatment (cotton seed oil extract) & 1303 & 18.88 & 24.24 \\
& 1304 & 19.72 & 24.83 \\
& 1305 & 20.25 & 25.28 \\
& Mean & 19.04 & 24.02 \\
& S.D. & 0.96 & 1.22 \\
& 1401 & 20.28 & 26.14 \\
& 1402 & 20.74 & 24.82 \\
& 1403 & 19.79 & 23.94 \\
& 1404 & 18.97 & 24.44 \\
& 1405 & 19.14 & 23.82 \\
& Mean & 19.78 & 24.63 \\
& S.D. & 0.75 & 0.93 \\
\hline
\end{tabular}

(2) Breeding condition: Stainless breeding box of the refining period and the test period [540 (W) $\mathrm{mm} \times 610(\mathrm{D}) \mathrm{mm} \times 200(\mathrm{H}) \mathrm{mm}$, MJ., Ltd.] was put in 5 rats bred on.

(3) Animal feed and drinking water: Feeds were fed free of SAFE 114 (Scientific Animal Food \& Engineering (SAFE), Route de Saint Bris-89290 Augy, France) containing radio-sterilized vitamin $\mathrm{C}$ and negative water using $\mathrm{R} / \mathrm{O}$ water.

5) Configuration and individual identification of test groups.

The individual identifications were indicated by individual numbers using saturated picric acid solution and individual identification cards were attached to the breeding boxes with the test number, sex, group number, individual number, duration of the test and the person responsible for the test.

6) Test methods.

(1) Primary induction (Intradermal induction induction): An intradermal injection the day before the three types of test substances following hair removal around the shoulder blade in the area of $4 \mathrm{~cm} \times 6 \mathrm{~cm}$, such as areas of the skin $(2 \mathrm{~cm} \times 4 \mathrm{~cm})$ was intradermally injected by $0.1 \mathrm{~mL}$.

(2) Secondary induction (local induction): After 6 days ( \pm 1 day) of primary intradermal injection, intradermal injection site was epilated and 10\% SLS (Sodium Lauryl Sulfate)/white vaseline was applied. On the next day, each test material was applied to sterile gauze $(2 \mathrm{~cm} \times 4 \mathrm{~cm})$, attached to the primary induction site of the animal, and stained with non-magnetic tape (Tegar- 
Table 15

Mortality and clinical signs

\begin{tabular}{|c|c|c|c|c|c|c|c|c|c|c|c|c|c|c|c|c|c|c|c|}
\hline \multirow[t]{2}{*}{ Group } & \multirow{2}{*}{$\begin{array}{l}\text { No. of } \\
\text { animals }\end{array}$} & \multirow[t]{2}{*}{ Mortality } & Animal number & \multicolumn{16}{|c|}{ Clinical signs } \\
\hline & & & Days & $\begin{array}{lllllllllll}0 & 1 & 2 & 3 & 4 & 5 & 6 & 7 & 8 & 9 & 1\end{array}$ & & 11 & 12 & 13 & 14 & 15 & 16 & 17 & 18 & 19 & 20 & 21 & 22 & 232 & 24 \\
\hline \multirow{5}{*}{$\begin{array}{l}\text { G1 negative } \\
\text { control } \\
\text { (sterile } \\
\text { saline) }\end{array}$} & 5 & $0 / 5^{\mathrm{a}}(0 \%)$ & 1101 & ---------- & - & - & - & - & - & - & - & - & - & - & - & - & - & - & - \\
\hline & & & 102 & --------- & - & - & - & - & - & - & - & - & - & - & - & - & - & - & - \\
\hline & & & 1103 & ---------- & - & - & - & - & - & - & - & - & - & - & - & - & - & - & - \\
\hline & & & 1104 & ---------- & - & - & - & - & - & - & - & - & - & - & - & - & - & - & - \\
\hline & & & 1105 & --------- & - & - & - & - & - & - & - & - & - & - & - & - & - & - & - \\
\hline \multirow{5}{*}{$\begin{array}{l}\text { G2 treatment } \\
\text { (sterile } \\
\text { saline) }\end{array}$} & 10 & $0 / 10(0 \%)$ & 1201 & ---------- & - & - & - & - & - & - & - & - & - & - & - & - & - & - & - \\
\hline & & & 1202 & --------- & - & - & _- & - & - & - & - & _- & _- & - & - & - & _- & - & - \\
\hline & & & 1203 & --------- & - & - & - & - & - & - & - & - & - & - & - & - & - & - & - \\
\hline & & & 1204 & --------- & - & - & - & - & - & - & - & - & - & - & - & - & - & - & - \\
\hline & & & 1205 & --------- & - & - & - & - & - & - & - & - & - & - & - & - & - & - & - \\
\hline \multirow{5}{*}{$\begin{array}{l}\text { G3 negative } \\
\text { control } \\
\text { (cotton } \\
\text { seed oil) }\end{array}$} & 5 & $0 / 5^{\mathrm{a}}(0 \%)$ & 1301 & --------- & - & - & - & - & - & - & - & - & - & - & - & - & - & - & - \\
\hline & & & $z$ & --------- & - & - & - & - & - & - & - & - & - & - & - & - & - & - & - \\
\hline & & & 1303 & --------- & - & - & - & - & - & - & - & - & - & - & - & - & - & - & - \\
\hline & & & 1304 & --------- & - & - & - & - & - & - & - & - & - & - & - & - & - & - & - \\
\hline & & & 1305 & --------- & - & - & - & - & - & - & - & - & - & - & - & - & - & - & - \\
\hline \multirow{10}{*}{$\begin{array}{l}\text { G4 treatment } \\
\text { (cotton } \\
\text { seed oil) }\end{array}$} & 10 & $0 / 10(0 \%)$ & 1401 & --------- & - & - & - & - & - & - & - & - & - & - & - & - & - & - & - \\
\hline & & & 1402 & --------- & - & - & - & - & - & - & - & - & - & - & - & - & - & - & - \\
\hline & & & 1403 & ---------- & - & - & - & - & - & - & - & - & - & - & - & - & - & - & - \\
\hline & & & 1404 & ---------- & - & - & - & - & - & - & - & - & - & - & - & - & - & - & - \\
\hline & & & 1405 & ---------- & - & - & - & - & - & - & - & - & - & - & - & - & - & - & - \\
\hline & & & & --------- & - & - & - & - & - & - & - & - & - & - & - & - & - & - & - \\
\hline & & & 14 & ---------- & - & - & - & - & - & - & - & - & - & - & - & - & - & - & - \\
\hline & & & 14 & -------- & - & - & - & - & - & - & - & - & - & - & - & - & - & - & - \\
\hline & & & 14 & --------- & - & - & - & - & - & - & - & - & - & - & - & - & - & - & - \\
\hline & & & 1410 & --------- & - & - & - & - & - & - & - & - & - & - & - & - & - & - & - \\
\hline
\end{tabular}

${ }^{\mathrm{a}}$ : No. of dead animals/No. of total animals, -: Normal.

derm, $3 \mathrm{M}$ ) and Corban (3 M) I kept it for a while.

(3) Cause sensitization: Sensitization second induction 14 days ( \pm 1 days), after depilation the flank of the animal left patch containing the test material $(2 \mathrm{~cm} \times 2 \mathrm{~cm})$, a non-irritating tape (Tegarderm, $3 \mathrm{M}$ ) and koban (Corban, $3 \mathrm{M}$ ) For 24 hours.

7) Progress observation.

(1) General symptom observation: General symptoms and presence or absence of dead animals were observed daily for all animals.

(2) Weight measurement: All animals were measured at the time of introduction, first induction day, second induction day, and sensitization day.

(3) Application site observation: After the induction of the test substance (for 24 hours), the patch at the application site was removed and the skin reaction was evaluated at 24 hours and 48 hours.

8) Assessment methods.

In the control group, the magnitude of the Magnuson and Cligman test is less than 1, and in the test group of 1 or more, it is generally considered to indicate sensation. If the control group shows grade 1 or higher, the response of the test group showing a more severe reaction considered to be due to sensitization. If the reaction is not clear, the material is subjected to induction to verify the results of the first test result. The test results are expressed as the frequency of the positive results of the test animals and the control animals. 
Table 16

Body weights (unit: g)

\begin{tabular}{|c|c|c|c|c|}
\hline Group & Animal number & First induction phase & Second induction phase & Challenge phase \\
\hline \multirow[t]{7}{*}{ G1 negative control (sterile saline) } & 1101 & 309.89 & 327.44 & 417.61 \\
\hline & 1102 & 333.87 & 358.21 & 447.56 \\
\hline & 1103 & 316.61 & 330.66 & 417.81 \\
\hline & 1104 & 337.15 & 353.37 & 425.45 \\
\hline & 1105 & 340.32 & 391.86 & 492.10 \\
\hline & Mean & 327.57 & 352.31 & 440.11 \\
\hline & S.D & 13.48 & 25.92 & 31.53 \\
\hline \multirow[t]{12}{*}{ G2 treatment (sterile saline) } & 1201 & 309.89 & 333.29 & 440.24 \\
\hline & 1202 & 320.98 & 365.25 & 432.66 \\
\hline & 1203 & 335.56 & 349.40 & 411.43 \\
\hline & 1204 & 335.33 & 368.79 & 416.53 \\
\hline & 1205 & 318.89 & 337.88 & 414.83 \\
\hline & 1206 & 326.35 & 366.59 & 401.43 \\
\hline & 1207 & 323.57 & 363.79 & 44.654 \\
\hline & 1208 & 335.59 & 378.17 & 442.85 \\
\hline & 1209 & 363.59 & 399.64 & 443.88 \\
\hline & 1210 & 314.17 & 368.85 & 432.27 \\
\hline & Mean & 328.39 & 363.17 & 428.27 \\
\hline & S.D & 15.30 & 19.31 & 15.96 \\
\hline \multirow{7}{*}{ G3 negative control (cotton seed oil) } & 1301 & 309.84 & 323.10 & 409.03 \\
\hline & 1302 & 325.35 & 370.74 & 447.08 \\
\hline & 1303 & 305.59 & 341.84 & 427.95 \\
\hline & 1304 & 336.21 & 366.96 & 417.46 \\
\hline & 1305 & 350.03 & 399.71 & 474.93 \\
\hline & Mean & 325.40 & 360.47 & 465.29 \\
\hline & S.D & 18.4 & 29.29 & 26.32 \\
\hline \multirow{12}{*}{ G4 treatment (cotton seed oil) } & 1401 & 317.60 & 355.92 & 412.42 \\
\hline & 1402 & 342.08 & 382.55 & 448.72 \\
\hline & 1403 & 350.20 & 417.88 & 481.60 \\
\hline & 1404 & 319.77 & 392.00 & 444.66 \\
\hline & 1405 & 311.98 & 350.08 & 460.98 \\
\hline & 1406 & 335.74 & 357.76 & 453.20 \\
\hline & 1407 & 342.28 & 359.87 & 442.27 \\
\hline & 1408 & 352.06 & 398.57 & 477.25 \\
\hline & 1409 & 332.47 & 399.08 & 485.34 \\
\hline & 1410 & 326.05 & 360.59 & 461.93 \\
\hline & Mean & 33.02 & 377.43 & 456.84 \\
\hline & S.D & 13.90 & 23.52 & 21.85 \\
\hline
\end{tabular}

\section{Results}

The following is a list of sequential results for the experiments in this study.

A. Aseptic test results: The aseptic test results are shown in Tables 9-11. When the liquid thioglycolic acid culture medium and the soybean casein digestion medium were cultured at the respective temperatures for 14 days, the microorganisms did not proliferate and the asepticity of the medium was confirmed. As a result of the performance test of the medium, the bacteria were clearly grown within 3 days and the fungi within 5 days when cultured at each temperature. When the specimens were inoculated into liquid thioglycolic acid medium and soybean casein digest medium and cultured at $(30 \sim 35)^{\circ} \mathrm{C},(20 \sim 25)^{\circ} \mathrm{C}$, no growth of bacteria was observed during total culture period. 
Table 17

Evaluation of skin response

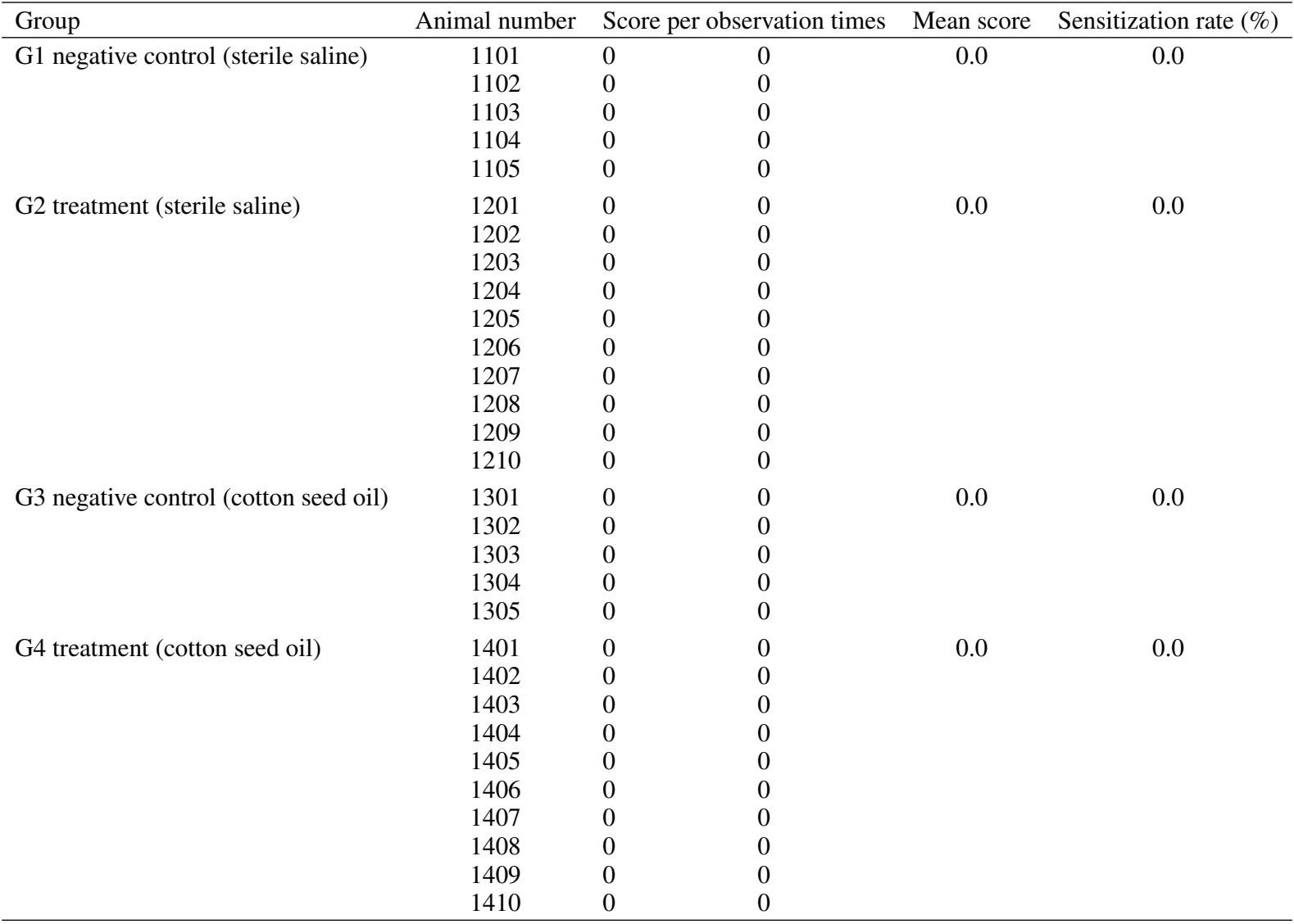

B. Cytotoxicity test results: The results of the qualitative analysis showing the morphological changes and the degree of dissolution of the cells are shown in Table 12. After incubation in the cells to which the eluate of the test substance was administered, a uniform monolayer was maintained but about $10 \%$ proliferation inhibition was observed. Therefore, the eluate of the test substance may have very slight reactivity to the cultured cells. In addition, quantitative analysis of the viable cell counts revealed that the effluent of the test material had a very slight effect on the number of viable cells (Table 12). The solvent control and negative control also did not show any toxicity to the cultured cells, and the positive control caused cytotoxicity in more than $75 \%$ of the total cells as expected.

C. Acute toxicity test results: Mortality and general symptoms Results No clinical signs and spermatozoa associated with the administration of the test substance were observed in all the treated animals for 4, 24, 48 and 72 hours after the injection (Table 13). In the case of weight change, normal weight change was observed in all test animals in the control group and the administration group on the day of administration and at 24, 48 and 72 hours after administration (Table 14).

D. Skin sensitization test results: No clinical signs or deaths due to the application of the test substance were observed during the skin sensitization test (Table 15). In the case of weight change, normal weight change was observed in both the test and control groups (Table 16). Skin responses were evaluated at 24 and 48 hours after the end of the test. Skin responses such as erythema and edema 
were not observed in the control group and the test group, and the solvent specific index was 0.0 and 0.0 , respectively (Table 17 ).

\section{Conclusions}

In this study, the safety of 4 Pin Multi-needle for meso-therapy was evaluated [16]. In the safety evaluation, sterility test, cytotoxicity test, acute toxicity test and skin sensitization test were carried out. The aseptic test was judged to be suitable for the aseptic test. In the cytotoxicity test, the MEM eluate of 4 Pin Multi-needle did not change the acidity of the culture medium, but showed very weak proliferation inhibition in mouse fibroblasts. Therefore, it is considered that the eluate of the test substance used in the cytotoxicity test has very slight cytotoxicity, and it is judged to be Grade 1 (Slightly cytotoxic) according to the criteria of the cytotoxicity of ISO 10993-5. The test substance also appears to meet the criteria for cytotoxicity in general medical devices that should be below grade 2 (Mild). The test results of the solvent control, negative control and positive control were as expected, demonstrating the effectiveness of the cytotoxicity test results. In the acute toxicity test, mortality, general symptoms, weight change and toxic response were evaluated within 72 hours after intravenous and intraperitoneal administration of $50 \mathrm{~mL} / \mathrm{kg} \mathrm{B}$.W. dose to ICR mice. After administration of the test substance, no deaths or specific clinical symptoms were observed, and normal weight changes were observed in all animals. From the above results, it was judged that ICR mice were treated with 4 Pin Multi-needle eluate at $50 \mathrm{~mL} / \mathrm{kg} \mathrm{B}$.W. for 72 hours and no systemic toxicity was observed. Finally, the skin sensitization test was conducted by inducing sensitization with a primary intradermal injection and a second transdermal application of sterile physiological saline and cottonseed oil extract to a Hartley guinea pig, and then evaluated the mortality, general symptoms, and skin sensitization induced by transdermal application. No deaths or abnormal findings were observed during the test period, and no skin reaction was observed in all test groups after 24 hours and 48 hours after the completion of sensitization. As a result, the 4 Pin Multineedle eluate in the Hartley guinea pig was evaluated as a weak skin sensitizer because it did not cause any skin reaction after sensitization exposure. In this study, the safety of 4 Pin Multi-needle was shown to be safe, and based on this, it is possible to provide safety and efficiency of operation and ease of operation for meso-therapy.

\section{Acknowledgments}

This study specifies that the results of the service test conducted by the Korea Testing and Research Institute (KTR), This work was supported by research fun of Catholic Kwandong University (CKURF201407130001), This work was supported by the Human Resource Training Program for Regional Innovation and Creativity through the Ministry of Education and National Research Foundation of Korea (NRF-2015H1C1A1035925).

\section{Conflict of interest}

None to report.

\section{References}

[1] Sugawara J, Kou S, Kolkubo K, Kuroda A, Hashizume Y, Kobayashi S, Maegawa J, Satake T. Application for lower 
facial fat reduction and tightening by static type monopolar $1-\mathrm{MHz}$ radio frequency for body contouring. Lasers in Surgery and Medicine 2017.

[2] Prikhnenko S. Polycomponent mesotherapy formulations for the treatment of skin aging and improvement of skin quality. Dove Medical Press (Open Access to Scientific and Medical Research) 2015; 7(8): 151-7.

[3] Kim S, Kye J, Lee M, Park B. Evaluation of mesotherapy as a transdermal drug delivery tool. Skin Research \& Technology 2016; 22(2): 158-63.

[4] Gazitaeva ZI, Drobintseva AO, Chung Y, Polyakova VO, Kvetnov IM. Cosmeceutical product consisting of biomimetic peptides: antiaging effects in vivo and in vitro. Clinical, Cosmetic and Investigationaal Dermatology 2017.

[5] Chen DL, Cohen JL, Green JB. Injectable agents affecting subcutaneous fats. Seminars in Cutaneous Medicine and Surgery 2015; 34(3): 134-7.

[6] El-Komy M, Hassan A, Tawdy A, Solimon M, Hady MA. Hair loss at injection sites of mesotherapy for alopecia. Journal of Cosmetic Dermatology 2017.

[7] Food and Drug Regulations in Korea Statute 2014-115, Common criteria for Biological Safety of Medical Devices.

[8] Food and Drug Regulations in Korea Statute 2014-155, Medical Device Standard.

[9] ISO 10993-12 (2012), Biological evaluation of medical devices - Part 12: Sample preparation and reference materials.

[10] ISO 10993-5 (2009), Biological evaluation of medical devices - Part 5: Tests for in vitro cytotoxicity.

[11] USP 36 (2013), <87> Biological reactivity tests, in vitro.

[12] ISO 10993-11 (2006), Acute Systemic Toxicity.

[13] ISO 10993-10 (2010), Gulnea pig maximization test (GMPT).

[14] ISO 10993-11(2006), Information on material-mediated pyrogens.

[15] USP 36, <151> Pyrogen Test.

[16] Phillips KS, Wang Y. FDA authors publihc articles on dermal filler materials, injection methods and skin preparation. Plastic and Reconstructive Surgery 2017. 